\title{
Suture repair of patellar inferior pole fracture: Transosseous tunnel suture compared with anchor suture
}

\author{
WENZHOU HUANG ${ }^{1-3}$, TIANLONG WU ${ }^{1-3}$, QIANGQIANG WEI ${ }^{1-3}$, \\ LONGHAI PENG ${ }^{1-3}$, XIGAO CHENG ${ }^{1-3}$ and GUICHENG GAO ${ }^{1-3}$ \\ ${ }^{1}$ Department of Orthopaedic Surgery, The Second Affiliated Hospital of Nanchang University; \\ ${ }^{2}$ Institute of Orthopedics of Jiangxi Province; ${ }^{3}$ Institute of Minimally Invasive Orthopedics of \\ Nanchang University, Nanchang, Jiangxi 330006, P.R. China
}

Received May 18, 2020; Accepted March 10, 2021

DOI: $10.3892 /$ etm.2021.10430

\begin{abstract}
Patellar inferior pole fracture is difficult to treat due to the inherent weakness of small comminuted distal fragments. However, suture fixation was recently introduced and reported. The aim of the present study was to evaluate and compare the clinical outcomes of two suture techniques, transosseous tunnel suture (TTS) and anchor suture (AS), for the fixation of patellar inferior pole fracture. A total of 35 patients with patellar inferior pole fracture treated at the Second Affiliated Hospital of Nanchang University (Nanchang, China) between June 2014 and April 2018 were retrospectively reviewed. Of these, 14 were treated with the TTS technique and 21 using AS fixation. The operation time, incision length and total cost were determined and compared. Functional outcomes were analyzed with the visual analog scale (VAS), Bostman and Lysholm scores and knee joint ranges of motion (ROMs). Postoperative complications were also observed and recorded. The mean follow-up was $22.6 \pm 9.7$ and $18.7 \pm 5.9$ months for TTS and AS, respectively. The groups were similar regarding age, sex, operative side and time to surgery. A smaller incision length and shorter operation time but higher hospital costs were observed in the AS group $(\mathrm{P}<0.01)$. For functional evaluation, there was no significant difference in VAS, Bostman and Lysholm scores or ROM between the 2 groups $(\mathrm{P}>0.05)$. No postoperative complications were observed in the TTS group. Only one patient in the AS group experienced a superficial minor wound infection. The TTS and AS techniques provided similarly satisfactory clinical outcomes for treating patellar inferior pole fracture. TTS had the advantage of cost-effectiveness due to saving anchors, while AS had a shorter operation time and a smaller incision length.
\end{abstract}

Correspondence to: Dr Guicheng Gao, Department of Orthopaedic Surgery, The Second Affiliated Hospital of Nanchang University, 1 Mingde Road, Nanchang, Jiangxi 330006, P.R. China

E-mail: gaoguicheng1983@163.com

Key words: patellar inferior pole fracture, fracture fixation, transosseous tunnel suture, anchor suture

\section{Introduction}

Inferior pole fracture of the patella is an extra-articular injury that accounts for $5 \%$ of all patellar fractures and usually requires operative treatment if displaced or associated with complete disruption of the extensor mechanism (1). However, displaced fracture fragments are typically small and comminuted and it is difficult to fix and maintain anatomical reduction (2). The inferior patellar pole is continuous with the patellar tendon and the key to reconstructing the fracture fragment surgically is to reestablish the extensor mechanism while simultaneously restoring articular congruency. The traditional treatment for displaced comminuted inferior pole fractures is partial patellectomy followed by repair of the patellar tendon. However, this treatment may potentially lead to a shorter long axis of the patella, which affects the functioning of the patellofemoral joint. Comparatively better outcomes have been achieved from fixation of displaced fragments $(3,4)$.

Most surgical methods use metallic fixation for inferior patellar pole fractures, such as tension band wiring (1), separate vertical wiring $(5,6)$, cannulated lag screws (7) and basket plates (8). Although these surgeries may ultimately achieve satisfactory outcomes, patients frequently require reoperation for implant removal or have soft-tissue irritation secondary to prominent hardware. Studies have reported on suture fixation to treat inferior patellar pole fractures, including transosseous tunnel suture (TTS) repair $(9,10)$ and anchor suture (AS) fixation $(11,12)$. Compared with using metal implants, suture fixation has similar functional outcomes but fewer hardware-related complications and lower implant removal rates. Sutures such as \#5 Ethibond and Fiberwire have been proven to be similar in strength to 18-gauge stainless steel wires $(13,14)$. Compared with transosseous techniques, AS offers the potential for minimal surgical dissection, no drilling and possibly reduced tissue trauma. However, to the best of our knowledge, no studies have compared the clinical outcomes of TTS with those of AS for inferior patellar pole fractures.

The purpose of the present study was to evaluate and compare the functional outcomes and complication rates in inferior patellar pole fractures treated with TTS and AS. It was hypothesized that patients would experience similar outcomes regardless of the fixation method. 


\section{Patients and methods}

Patients. The present study provided a retrospective analysis of patients with sustained patellar inferior pole fracture treated at the Department of Orthopedics of the Second Affiliated Hospital of Nanchang University (Nanchang, China) between June 2014 and April 2018. The inclusion criteria were the use of TTS or AS for fracture fixation, age of $>18$ years and a minimum follow-up of at least 12 months. The exclusion criteria were open fractures, concomitant knee fractures other than those of the patella, multiple traumata, acute infection, osteoarthritis, rheumatoid arthritis or musculoskeletal disorders.

The present study was approved by the Ethics Committee of the Second Affiliated Hospital of Nanchang University (Nanchang, China) and all patients or their relatives provided written informed consent prior to inclusion. A total of 14 patients ( 9 males and 5 females; mean age, 47.6 years; age range, 32-70 years) with patellar inferior pole fracture who underwent TTS and 21 patients (12 males and 9 females; mean age, 45.6 years; age range, 25-68 years) who underwent AS were enrolled in the present study. The 2 groups were comparable in terms of age, sex and operative side. The condition of the soft tissue was carefully assessed prior to surgery to understand the optimal timing of the operation. The group treated with TTS underwent surgery at an average of 3.6 days after trauma (range, 3-6 days) and the AS group underwent surgery at an average of 4.0 days (range, 2-8 days) after trauma.

Surgical techniques. After general anesthesia or lumbar anesthesia, the patient was placed in a supine position on the operating table with tourniquet inflation. All patients received antibiotics (first-generation cephalosporin) $30 \mathrm{~min}$ prior to surgery. A total of three surgeons from the same group at our department performed all surgeries together. A midline longitudinal skin incision on the knee was made. The distal pole patella fragment was identified, and hematoma and soft tissue from the fracture site were removed. The proximal fracture edges were irrigated with saline and debrided with a curette and rongeur to promote fracture healing. With regard to the TTS technique, the surgery was performed similar to the example of Swensen et al (10). A total of three transpatellar tunnels were drilled inferior to superior using a $2.5-\mathrm{mm}$ drill bit or pin at the central, medial and lateral proximal patellar sites. Next, \#5 Ethibond (Ethicon) braided polyester sutures were used in Krackow locking fashion up and down the medial and lateral edges of the patellar tendon. The suture limbs were passed through the central, medial and lateral tunnels using a suture passer. The fracture was next reduced with bone reduction forceps and C-arm fluoroscopy indicated good fracture reduction. The sutures were then tied at the superior pole with the knee in extension. For the AS group, two holes were drilled into the medial and lateral proximal fracture edges and suture anchors (4.5 mm Healix Advance anchor; DePuy Mitek, Inc.) with two Orthocord sutures were placed into each of the holes. After reduction with bone reduction forceps and verification with $\mathrm{C}$-arm fluoroscopy, the sutures from each anchor were passed through the medial and lateral sides of the patellar tendon in a horizontal Krackow locking fashion. In addition, tears observed in the retinaculum were repaired and after subcutaneous layer closure, the skin layer was closed with running subcuticular sutures. The operation time, incision length and total cost were determined and recorded for comparison between groups.

Rehabilitation protocol. In all patients, the knee was immobilized at $0^{\circ}$ postoperatively with an orthesis or plaster for 4 weeks to allow for healing of the retinacular tears and patellar ligament to the bone. The patients were allowed to begin partial weight-bearing at 50\% of their weight at 2 weeks postoperatively, with full weight by 3-4 weeks. Both active and passive range of motion (ROM) exercises were started at the fourth week postoperatively with the goal of $90^{\circ}$ flexion for the next 2 weeks and full ROM was allowed from the sixth week postoperatively.

Clinical assessment. The patients were evaluated both radiologically and clinically at 3,6 and 12 months after the surgery and then until the last follow-up. Antero-posterior and lateral radiographs were obtained to assess reduction quality and bone union. The degree of pain was assessed using the visual analog scale (VAS) (15) ranging from 0-10. Knee functional outcomes were evaluated using the Bostman et al (16) and Lysholm and Gillquist (17) scoring systems. The final knee-joint ROM was measured. In addition, postoperative complications, including nonunion, implant failure and infection, were recorded.

Statistical analysis. Statistical analyses were performed using SPSS statistical software 23.0 (IBM Corp.). Continuous variables are presented as the mean \pm standard deviation and categorical variables were expressed as $\mathrm{n}(\%)$. The Kolmogorov-Smirnov test was used to check the data for normality of distribution. The t-test was employed to compare variables with a normal distribution. The nonparametric Mann-Whitney U-test was used for variables without a normal distribution, such as the ROM and VAS score. Differences in categorical variables between the two groups were assessed with Fisher's exact test. $\mathrm{P}<0.05$ was considered to indicate statistical significance.

\section{Results}

General data. No significant differences in age, sex, operative side or time to surgery were present between the two groups. 14 patients in the TTS group and 21 in the AS group attained their final follow-ups. The mean follow-up duration was $22.6 \pm 9.7$ months in the TTS group and $18.7 \pm 5.9$ months in the AS group ( $\mathrm{P}=0.191$; Table I).

Surgical conditions. The average operation time was $62.3 \pm 6.4 \mathrm{~min}$ in the TTS group and $41.1 \pm 4.2 \mathrm{~min}$ in the AS group $(\mathrm{P}<0.01)$. The mean incision length was significantly smaller in the AS group than in the TTS group $(8.3 \pm 1.1$ vs. $12.4 \pm 1.2 \mathrm{~cm}, \mathrm{P}<0.01)$. However, the AS group incurred higher hospital costs than the TTS group ( $¥ 30,856 \pm 859$ vs. $20,778 \pm 489, \mathrm{P}<0.01$; Table I).

Functional outcomes. To evaluate functional outcomes in the present study, the Bostman and Lysholm scoring systems were utilized. At the last follow-up, the mean Bostman score was $28.7 \pm 1.3$ (excellent) in the group treated with TTS and 
Table I. Characteristics of the two groups.

\begin{tabular}{lccr}
\hline Variable & TTS group $(\mathrm{n}=14)$ & AS group $(\mathrm{n}=21)$ & P-value \\
\hline Age (years) & $47.6 \pm 10.6$ & $45.6 \pm 11.5$ & $0.602^{\mathrm{a}}$ \\
Sex (male/female) & $9(64) / 5(36)$ & $12(57) / 9(43)$ & $0.737^{\mathrm{b}}$ \\
Side (left/right) & $6(43) / 8(57)$ & $7(33) / 14(67)$ & $0.724^{\mathrm{b}}$ \\
Time to surgery (days) & $3.6 \pm 0.9$ & $4.0 \pm 1.5$ & $0.606^{\mathrm{c}}$ \\
Follow-up (months) & $22.6 \pm 9.7$ & $18.7 \pm 5.9$ & $0.191^{\mathrm{a}}$ \\
Operation time (min) & $62.3 \pm 6.4$ & $41.1 \pm 4.2$ & $<0.01^{\mathrm{a}}$ \\
Incision length (cm) & $12.4 \pm 1.2$ & $8.3 \pm 1.1$ & $<0.01^{\mathrm{c}}$ \\
Total cost (¥) & $20,778 \pm 489$ & $30,856 \pm 859$ & $<0.01^{\mathrm{a}}$
\end{tabular}

${ }^{\mathrm{a}}$-test, ${ }^{\mathrm{b}}$ Fisher's exact test, ${ }^{\mathrm{C}}$ Mann-Whitney U-test. Values are expressed as $\mathrm{n}(\%)$ or the mean \pm standard deviation. $¥$, Chinese currency unit, yuan; TTS, transosseous tunnel suture; AS, anchor suture.

Table II. Inter-group comparison of functional outcomes.

\begin{tabular}{lcrr}
\hline Variable & TTS group & AS group & P-value \\
\hline ROM, ${ }^{\circ}$ (extension/flexion) & $0 / 133.6 \pm 6.3$ & $0 / 129.8 \pm 6.2$ & 0.089 \\
VAS score & $1.6 \pm 0.9$ & $1.4 \pm 0.9$ & 0.654 \\
Bostman score & $28.7 \pm 1.3$ & $27.8 \pm 1.4$ & 0.071 \\
Lysholm score & $94.4 \pm 3.9$ & $91.4 \pm 5.1$ & 0.118 \\
Complications & None & 1 infection & - \\
\hline
\end{tabular}

All statistical comparisons were performed with the Mann-Whitney U-test. Values are expressed as n (\%) or the mean \pm standard deviation. TTS, transosseous tunnel suture; AS, anchor suture; ROM, range of motion; VAS, visual analogue scale.

$27.8 \pm 1.4$ (good) in the group who received AS. Furthermore, the mean Lysholm score at the final follow-up was $94.4 \pm 3.9$ (good) in the TTS group and 91.4 \pm 5.1 (good) in the AS group. Differences in the Bostman and Lysholm scores were not statistically significant between the groups $(\mathrm{P}=0.071$ and $\mathrm{P}=0.118$, respectively). Pain evaluation using the VAS score also indicated no significant differences between the TTS group and the AS group (1.6 \pm 0.9 vs. $1.4 \pm 0.9, \mathrm{P}=0.654)$. At the time of the final follow-up, the mean knee joint ROM was $133.6 \pm 6.3^{\circ}$ in the TTS group and $129.8 \pm 6.2^{\circ}$ in the AS group, with no significant differences in ROM between the two groups $(\mathrm{P}=0.089$; Table II $)$.

Radiographic results and Complications. Radiographic signs of bone union were achieved after approximately 3 months in both groups (Figs. 1 and 2). No postoperative complications, such as infection, nonunion or implant failure, were observed in the TTS group. However, one patient in the AS group had a superficial minor wound infection, which was successfully treated by daily dressing changes and adapted antibiotics. No cases of deep infection occurred. Furthermore, none of the patients in either group had any skin irritation or reduction loss (Table II).

\section{Discussion}

Fracture of the inferior patellar pole is characterized by avulsion of the patellar tendon (18); thus, repairing the extensor mechanism and stable fixation are vital for osteosynthesis of the inferior pole. The tension-band-wiring technique has been the most commonly used method for displaced patellar fracture, but it is difficult to implement for inferior pole fixation due to the inherent weakness of small comminuted distal fragments. Other surgical techniques, such as separate vertical wiring or basket plate and their modifications, may provide stable fixation and satisfactory results $(2,5,8,19,20)$. However, this specific plate is not available in numerous institutions and the prominent wire knots or the relative bulk of the plate covered with thin soft tissue may cause skin irritation in the flexed knee position, possibly necessitating metal implant removal. Various studies have reported on the use of nonmetallic implants for fixation of inferior pole fractures, such as nonabsorbable braided sutures $(9,10,21,22)$ and suture anchors $(11,12)$.

Egol et al (9) demonstrated that suture repair had fewer hardware-related postoperative complications, with only one-quarter of the reoperation rate of standard tension band fixation. Recently, a retrospective study of surgically treated patellar fractures was performed in which suture fixation resulted in a lower number and rate of soft-tissue irritation and reoperation rate compared with hybrid and metal fixation, even though these advantages were negated with the addition of a metal tension band wire (22). Furthermore, a prior study reported a hardware removal rate of $\sim 52 \%$ following the use of metallic implants (23). In the present study, both TTS and 

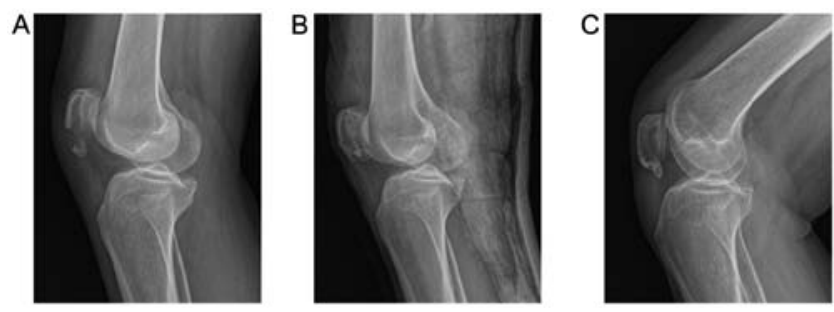

Figure 1. Lateral knee radiographs of the transosseous tunnel suture group. (A) Preoperative X-ray film. (B) Postoperative X-ray. (C) At one year follow-up X-ray.
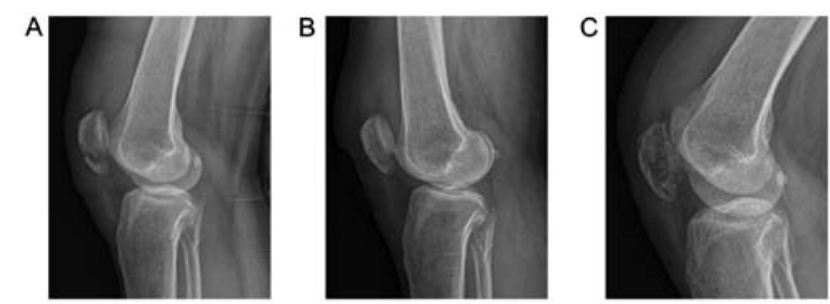

Figure 2. Lateral knee radiographs of the anchor suture group. (A) Preoperative X-ray film. (B) Postoperative X-ray. (C) At one year follow-up X-ray.

AS techniques resulted in positive clinical outcomes with low implant-related complication rates. Only one of 21 patients in the AS group had a superficial minor wound infection and none of the patients complained of any symptomatic hardware or required implant removal.

The major concern in suture replacement of stainless steel wire for the treatment of patellar fracture is suture fixation strength. To address this, previous biomechanical studies have verified the fixation properties of sutures in the tension band technique $(13,14,24)$. Patel et al $(13)$ reported that the \#5 Ethibond braided polyester suture is an alternative to stainless steel wire for fixation of patellar fractures. In addition, a study evaluated the stiffness and failure strength of Fiberwire, a braided polyblend suture, using a three-point-bend model, and indicated that double-strand Fiberwire has a significantly higher failure load than stainless steel wire (14). This was also confirmed in clinical studies; 4 studies comprising 29 patients demonstrated a convincing clinical outcome using Ethibond or Fiberwire sutures for the treatment of inferior pole patella fractures. In the present study, \#5 Ethibond was used for the TTS technique and acceptable clinical outcomes were achieved $(9,10,21,22)$.

Suture anchors have been used extensively for numerous tendon and ligament repairs (25-27), and the technique using two suture anchors for the treatment of patellar inferior pole fractures was recently introduced $(11,12)$. Compared with the TTS technique for patellar inferior pole fracture, the AS technique is a more convenient surgical procedure, given the requirement to expose only the distal pole of the patella without transosseous drilling. Minimal surgical dissection may be associated with a shorter operative time, less soft-tissue trauma and a lower risk of iatrogenic patellar articular cartilage. Although the fixation strength of AS in the treatment of patellar fractures has not been proven experimentally, a recent study evaluated the use of suture anchor fixation for patellar tendon ruptures and reported that the suture anchor yields biomechanical effects similar to those of transosseous suture repair (28). It is recommended that bone anchors are held through intact cortex bone in tendon repairs. However, with inferior pole fragment avulsion, bone anchors must be inserted into cancellous bone without using the rim of the cortex, which may constitute a weak point leading to implant failure. Kadar et al (12) reported 2 cases (7.4\%) of implant failure in 27 patients with distal pole patellar fracture who underwent the AS technique. In both cases, the anchor was pulled out of the main patellar fragment. Regardless, such failure did not occur in the study by Anand et al (11) or the present study.

On the basis of the results of the present study, it was indicated that AS has comparable functional outcomes, pain levels and knee-joint ROM to TTS. Along with the benefits of a simpler procedure and less extensive exposure, the operation time and surgical incision were significantly shorter for the AS group than for the TTS group. A disadvantage of the AS technique is its increased cost: In the present study, the hospital costs for the AS group were significantly higher than those for the TTS group, mostly because the cost for two suture anchors is $¥ 10,000$ at our institution.

The limitations of the present study are its retrospective nature and small sample size. Non-randomized controlled trials on patients with orthopedic trauma do not necessarily allow for the identification of factors influencing implant selection, which may lead to inherent selection bias with a clear potential to influence outcomes. Another limitation is that two different suture types were used for each group of repairs. TTS was performed with \#5 Ethibond, whereas Orthocord was used for AS, potentially causing instrument bias in the study. Further biomechanical studies and randomized investigations are warranted in the future.

In conclusion, TTS and AS provide similar satisfactory clinical outcomes for treating patellar inferior pole fracture and complication rates are low with both techniques. TTS has the advantage of cost-effectiveness by saving anchors and AS is associated with reduced operation time and a smaller incision length. Thus, both the TTS and AS techniques are acceptable treatment methods for patellar inferior pole fracture.

\section{Acknowledgements}

Not applicable.

\section{Funding}

The present study was supported by the Science and Technology Program of Jiangxi Educational Commission (grant no. GJJ200246).

\section{Availability of data and materials}

The datasets used and/or analyzed during the current study are available from the corresponding author on reasonable request.

\section{Authors' contributions}

GCG and XGC conceived and designed the study. WZH and TLW analyzed the data and edited the manuscript. 
QQW and LHP helped to perform the follow-up and collect patients' data. GCG and XGC confirm the authenticity of all the raw data. All authors read and approved the final manuscript.

\section{Ethical approval and consent to participate}

The present study was approved by the Ethics Committee of the Second Affiliated Hospital of Nanchang University [Nanchang, China; reference no. 2018 (026)]. Written informed consent was obtained from the patients or their relatives.

\section{Patient consent for publication}

Consent for publication of the patients' data and images was obtained from patients or their relatives.

\section{Competing interests}

The authors declare that they have no competing interests.

\section{References}

1. Yang X, Wu Q, Lai CH and Wang X: Management of displaced inferior patellar pole fractures with modified tension band technique combined with cable cerclage using Cable Grip System. Injury 48: 2348-2353, 2017.

2. Oh HK, Choo SK, Kim JW and Lee M: Internal fixation of displaced inferior pole of the patella fractures using vertical wiring augmented with Krachow suturing. Injury 46: 2512-2515, 2015.

3. Veselko M and Kastelec M: Inferior patellar pole avulsion fractures: Osteosynthesis compared with pole resection Surgical technique. J Bone Joint Surg Am 87 (Suppl 1): S113-S121, 2005.

4. Matejcic A, Puljiz Z, Elabjer E, Bekavac-Beslin M and Ledinsky M: Multifragment fracture of the patellar apex: Basket plate osteosynthesis compared with partial patellectomy. Arch Orthop Trauma Surg 128: 403-408, 2008.

5. He S, Huang X, Yan B, Zhu J, Bao N and Zhao J: Modified technique of separate vertical wiring for the fixation of patellar inferior pole fracture. J Orthop Trauma 32: e145-e150, 2018.

6. Song HK, Yoo JH, Byun YS and Yang KH: Separate vertical wiring for the fixation of comminuted fractures of the inferior pole of the patella. Yonsei Med J 55: 785-791, 2014.

7. Chang SM and Ji XL: Open reduction and internal fixation of displaced patella inferior pole fractures with anterior tension band wiring through cannulated screws. J Orthop Trauma 25: 366-370, 2011

8. Kastelec M and Veselko M: Inferior patellar pole avulsion fractures: Osteosynthesis compared with pole resection. J Bone Joint Surg Am 86: 696-701, 2004.

9. Egol K, Howard D, Monroy A, Crespo A, Tejwani N and Davidovitch R: Patella fracture fixation with suture and wire: You reap what you sew. Iowa Orthop J 34: 63-67, 2014.

10. Swensen S, Fisher N, Atanda A and Egol KA: Suture repair of a pole patella fracture. J Orthop Trauma 31 (Suppl 3): S28-S29, 2017.

11. Anand A, Kumar M and Kodikal G: Role of suture anchors in management of fractures of inferior pole of patella. Indian J Orthop 44: 333-335, 2010
12. Kadar A, Sherman H, Drexler M, Katz E and Steinberg EL: Anchor suture fixation of distal pole fractures of patella: Twenty seven cases and comparison to partial patellectomy. Int Orthop 40: 149-154, 2016

13. Patel VR, Parks BG, Wang Y, Ebert FR and Jinnah RH: Fixation of patella fractures with braided polyester suture: A biomechanical study. Injury 31: 1-6, 2000.

14. Wright PB, Kosmopoulos V, Coté RE, Tayag TJ and Nana AD: FiberWire is superior in strength to stainless steel wire for tension band fixation of transverse patellar fractures. Injury 40: 1200-1203, 2009.

15. Castrejón I, Chua JR and Pincus T: A RheuMetric physician checklist to quantitate levels of inflammation, damage and distress on 0-10 visual analogue scales. Clin Exp Rheumatol 35 (Suppl 107): S21-S25, 2017.

16. Bostman O, Kiviluoto O and Nirhamo J: Comminuted displaced fractures of the patella. Injury 13: 196-202, 1981.

17. Lysholm $\mathbf{J}$ and Gillquist $\mathbf{J}$ : Evaluation of knee ligament surgery results with special emphasis on use of a scoring scale. Am J Sports Med 10: 150-154, 1982.

18. Massoud EIE: Repair of comminuted fracture of the lower patellar pole. Ulus Travma Acil Cerrahi Derg 23: 150-155, 2017.

19. Matejčić A, Ivica M, Jurišić D, Cuti T, Bakota B and Vidović D: Internal fixation of patellar apex fractures with the basket plate: 25 years of experience. Injury 46 (Suppl 6): S87-S90, 2015.

20. Huang HC, Su JY and Cheng YM: Modified basket plate for inferior patellar pole avulsion fractures: A report of three cases. Kaohsiung J Med Sci 28: 619-623, 2012.

21. Camarda L, La Gattuta A, Butera M, Siragusa F and D'Arienzo M: FiberWire tension band for patellar fractures. J Orthop Traumatol 17: 75-80, 2016.

22. Shea GK, Hoi-Ting So K, Tam KW, Yee DK, Fang C and Leung F: Comparing 3 different techniques of patella fracture fixation and their complications. Geriatr Orthop Surg Rehabil 10: $2151459319827143,2019$.

23. LeBrun CT, Langford JR and Sagi HC: Functional outcomes after operatively treated patella fractures. J Orthop Trauma 26: 422-426, 2012.

24. McGreal G, Reidy D, Joy A, Mahalingam K and Cashman WF: The biomechanical evaluation of polyester as a tension band for the internal fixation of patellar fractures. J Med Eng Technol 23: 53-56, 1999.

25. Gaines RJ, Grabill SE, DeMaio M and Carr D: Patellar tendon repair with suture anchors using a combined suture technique of a Krackow-Bunnell weave. J Orthop Trauma 23: 68-71, 2009.

26. Petri M, Dratzidis A, Brand S, Calliess T, Hurschler C, Krettek C, Jagodzinski M and Ettinger M: Suture anchor repair yields better biomechanical properties than transosseous sutures in ruptured quadriceps tendons. Knee Surg Sports Traumatol Arthrosc 23: 1039-1045, 2015.

27. Derwin KA, Sahoo S, Zajichek A, Strnad G, Spindler KP, Iannotti JP and Ricchetti ET: Tear characteristics and surgeon influence repair technique and suture anchor use in repair of superior-posterior rotator cuff tendon tears. J Shoulder Elbow Surg 28: 227-236, 2019.

28. Sherman SL, Black B, Mooberry MA, Freeman KL, Gulbrandsen TR, Milles JL, Evans L, Flood D and Pfeiffer F: Biomechanical evaluation of suture anchor versus transosseous tunnel patellar tendon repair techniques. J Knee Surg 32: $825-832,2019$.

(i) 1 This work is licensed under a Creative Commons Attribution-NonCommercial-NoDerivatives 4.0 International (CC BY-NC-ND 4.0) License. 